Stoa

Vol. 3, no. 6, 2012, pp. 101-117

ISSN 2007-1868

\title{
UNA PROPUESTA PARA EL REALISMO ESTRUCTURAL
}

\author{
Thomas Meier \\ Ludwig-Maximilians Universität \\ Facultad de Filosofía, \\ Filosofía de la Ciencia \\ y Ciencia de la Religión
}

RESUMEN: El propósito de este trabajo es motrar una nueva forma de aproximarse al realismo estructural. Esta postura se ha visto criticada en la literatura reciente. Se mencionan los dos argumentos que han llevado a la formulación de realismo estructural dentro del debate del realismo científico, la "tesis nomilagro" y la "meta-inducción pesimista". Se introduce el marco conceptual de la metateoría estructuralista para argumentar que, una vez adoptado este marco conceptual, es posible responder a las críticas y clarificar el realismo estructural, especialmente la noción de continuidad estructural.

PALABRAS ClAVE: Realismo estructural · "tesis no-milagro" • "meta-inducción pesimista" · metateoría estructuralista $\cdot$ continuidad estructural

ABST RACT: The purpose of this work is to outline a new approach to the debate on structural realism. This position has been criticized in the recent literature. First, the two arguments which led to the introduction of structural realism into the debate on scientific realism are introduced, namely, the "no-miracles Second, argument" the and framework the of "pessimistic structuralist metainduction". meta-theory is introduced in order to argue as follows. Once this framework is adapted, it is possible to respond to the critiques that have been made to structural realism and to clarify this position, especially the notion of structural continuity.

KEYwords: Structural realism . "no-miracle argument" · "pessimistic metainduction" · stucturalist meta-theory · structural continuity 


\section{Introducción}

Este trabajo tiene como principal motivación contribuir a la discusión sobre el realismo estructural en epistemología y en filosofía de la ciencia general.

Desde la publicación del artículo de John Worrall en 1989, se ha generado un debate amplio sobre el realismo estructural. La posición del realismo estructural, expuesta por Worrall, afirma lo siguiente: "Cuando nuestras teorías empíricas cambian en el transcurso del tiempo, lo que sí es retenido es el contenido estructural de nuestras teorías. La estructura matemática de nuestras teorías es lo que se retiene y lo que es continuo, aún en casos de cambio teórico radical”. Así, afirma Worrall, hay continuidad estructural entre teorías radicalmente diferentes. Concluyentemente, las partes estructurales de nuestras teorías no se abandonan porque son correctas, mientras otros componentes de las teorías, como los nombres de los referentes, cambian normalmente.

En este trabajo, la propuesta es contribuir a una clarificación de la postura original del realismo estructural, proponiendo un marco conceptual adecuado para ello. Este marco será el de la metateoría estructuralista. En la propuesta de Worrall se ha aludido a la continuidad estructural en el siguiente sentido: la reaparición de ciertas ecuaciones diferenciales en una teoría sucesora. La propuesta en este trabajo es mostrar que un enfoque tal no puede abarcar los cambios estructurales que ocurren en todas las disciplinas científicas. Se sugiere que no sólo las ecuaciones pueden reaparecer o ser retenidas cuando ocurre un cambio teórico radical. Para ello, argumentaré que la concepción de lo que entendemos por una teoría empírica debe ser enfocada desde un punto de vista modelo-teórico. Sólo de esta manera se obtiene la estructura lógica de nuestras teorías empíricas de manera exhaustiva.

En los últimos años, se han dado varios desarrollos en la discusión sobre el realismo estructural. Se han distinguido dos tipos de concepción, el realismo estructural epistémico y el realismo estructural óntico. La primera de estas dos posturas afirma que todo nuestro conocimien-

to es de forma estructural y que no se puede conocer las propiedades de las entidades que son parte de estructuras. La versión óntica es aún más fuerte al afirmar que todo lo que existe es estructural, que los 
objetos solamente llenan espacios dentro de estructuras y que no tienen existencia por sí misma. Para el realismo estructural óntico, las entidades ontológicamente básicas son estructuras. Esta concepción es expuesta de manera sistemática en Ladyman y Ross (2007), y se encuentra principalmente motivada por la física contemporánea.

Este trabajo enfatiza en un aporte a la versión epistémica del realismo estructural. Argumentaré que, si se adopta el marco conceptual de la metateoría estructuralista -que es una de las concepciones modeloteóricas-, se abre un camino para formular una aproximación más comprometedora al realismo estructural. Examinaré los postulados del mismo desde la perspectiva de la metateoría estructuralista, esto para mostrar cómo se clarifica y fortalece el realismo estructural si aplicamos nociones técnicas del marco conceptual del estructuralismo, En este sentido, este trabajo es una propuesta programática.

Para ello, comenzaré por una detallada exposición del realismo estructural, y mostraré los dos argumentos que llevaron a su reintroducción en el debate actual sobre el realismo científico. Estos, son ampliamente conocidos como la "meta inducción pesimista" (MIP), de Larry Laudan y "la tesis del no-milagro" (TNM), por parte de Smart, Boyd y Putnam, entre otros. El primero es un argumento que intenta refutar el realismo científico, refiriendose a la historia de la ciencia. El segundo lo defiende.

Después mostraré que el estructuralismo nos ofrece todas las herramientas formalies para modelar lo que Worrall llama "continuidad estructural" y lo que parece ser la noción central en el realismo estructural. Las nociones técnicas de la metateoría estructuralista juegan un rol central en este paso.

\section{La "tesis del no-milagro" y la "meta-inducción pesimista"}

En la discusión sobre el realismo científico, la "tesis del no-milagro" es conocida como una defensa contundente del realismo. El argumento ha sido formulado ya en distintas ocasiones por Smart (1963, 1979), Putnam (1975) y Boyd (1983).

Hay distintas maneras de formular este argumento. Pienso que la siguiente formulación es la más clara posible, aparte de ser dedutivamente valida: 
(I) Nuestras teorías empíricas son exitosas;

(II) Si nuestras teorías empíricas son exitosas y falsas, entonces es un milagro que nuestras teorías empíricas sean exitosas;

(III) No es un milagro que nuestras teorías empíricas sean exitosas;

(IV) Por lo tanto, nuestras teorías empíricas son verdaderas.

Formulado así, la "tesis del no-milagro" es un argumento válido. Siguiendo a este argumento, se muestra que el realismo científico puede ser defendido exitosamente. Para llegar a comprender la introducción de Worrall del realismo estructural, pasemos a la introducción del argumento antirrealista que ha podido debilitar el realismo científico de forma contundente, la así llamada "meta-inducción pesimista", formulada por Laudan (1981). Esta propuesta obtiene fuerza a partir de la referencia a la historia de la ciencia. Laudan mostró que, si se toma en cuenta el desarrollo fáctico de la ciencia, no se puede afirmar que haya habido una aproximación acumulativa a la verdad, donde las teorías sucesoras siempre son más cercanas a la verdad que sus antecesores. Así, Laudan nos dice que el éxito empírico de una teoría no conlleva su verdad aproximativa. Su argumento puede ser formulado de lo siguiente:

(I) En el transcurso de la historia, todas nuestras teorías empíricas han salido como falsas. Siempre han sido abandonadas o reemplazadas por teorías sucesoras;

(II) Si éste siempre ha sido el caso en el pasado, al menos es muy probable que así será para las teorías actuales y futuras que hoy día tomamos como verdaderas;

(III) Por lo tanto, es muy probable que nuestras teorías empíricas actuales no sean verdaderas, y que las futuras continuen erradas.

Siguiendo a Laudan, no es justificable decir que nuestras teorías empíricas son verdaderas. Es aún más probable asumir que sean falsas y por esto, el realismo científico no puede ser sostenido. La introducción de la posición de Worrall está situada en este contexto. Worrall 
intenta incorporar la "tesis del no-milagro" y la "meta-inducción pesimista", para proponer una versión de realismo científico menos fuerte, el realismo estructural.

\section{Realismo estructural - la noción de continuidad estructural}

Aunque el realismo estructural como posición epistemológica puede ser encontrado ya en Poincaré (1912), Russell (1912, 1927), Carnap (1928) y otros, fue Worrall (1989) quien lo introdijo en el debate moderno sobre el realismo científico, él combina la propuesta epistemológica de Poincaré con una posición sobre cambio teórico. Queriendo sacar lo mejor de TNM y de MIP, muestra tanto que nuestras teorías son verdaderas porque son exitosas, como, también, que nuestras teorías sufren cambios (a veces radicales) en el transcurso de la historia. Según Worrall, mientras los nombres de las entidades que son parte de nuestras teorías cambian totalmente, permanecen ciertas estructuras matemáticas durante tal cambio. De estas estructuras podemos tener conocimiento.

En su trabajo original, Worrall fundamenta su posición en un estudio de caso de la óptica, el cambio de la óptica de Fresnel a la de Maxwell. La continuidad estructural, identificada por Worrall, se manifiesta en que ciertas ecuaciones diferenciales que forman parte de la teoría de Fresnel, también aparecen en la teoría de Maxwell; en cambio, el término "éter" ya no aparece en la óptica de Maxwell, donde se introduce el campo electromagnético.

A pesar de que Fresnel estaba bastante mal sobre qué es lo que oscila, estaba correcto, desde el punto de vista posterior, no sólo sobre los fenómenos ópticos, sino también que estos fenómenos dependen de las oscilaciones de algo que en un ángulo recto con la luz. Así que, si nos restringimos al nivel de las ecuaciones matemáticas - no notando el nivel fenomenal- hay de heco continuidad completa entre las teoróas de Fresnel y de Maxwell (Worrall, 1989, pp. 118-119). ${ }^{1}$

Lo que Worrall afima es que nuestras teorías empíricas no son totalmente reemplazadas cuando cambian, sus partes estructurales se retienen. Lo que es de importancia central es que para el realismo estruc-

${ }^{1}$ Todas las citas en este artículo han sido traducidas del inglés o del alemán por el autor del mismo. 
tural no tenemos acceso epistémico a los referentes de las entidades inobservables que aparecen en nuestras teorías. En cambio, sí es posible tener conocimiento de las estructuras matemáticas -ecuacionesdentro de las cuales aparecen estas entidades. Si ocurre que las mismas ecuaciones son parte de teorías radicamente diferentes, es razonable asumir que lo que representa el mundo de la mejor manera son estas mismas.

Hay dos presunciones metafísicas centrales en el realismo estructural:

(I) Lo que sobrevive cambios teóricos radicales es lo que realmente está aferrado al mundo. Si ciertas estructuras son retenidas durante un cambio teórico, es razonable afirmar que la persistencia de estas estructuras refleja en cada caso específico una parte específica del mundo.

(II) La persistencia de ciertas estructuras durante algún cambio teórico hace razonable asumir que al menos la parte estructural de nuestras teorías empíricas representa el mundo correctamente. Por lo tanto, podemos decir que lo que conocemos del mundo son las estructuras mediante las cuales obtenemos una representación correcta del mismo.

\section{Objeciones al realismo estructural}

La propuesta de Worrall llevó a una serie de objeciones, las cuales han sido presentadas de la manera más representativa por Psillos (1999, pp 146-161). La siguiente lista expone estas objeciones:

(I) El realismo estructural debe ser distanciado más claramente del antirrealismo. Sólo apelar a la idea de la continuidad en el nivel de las ecuaciones matemáticas no basta, ya que también es combinable con el instrumentalismo.

(II) No es claro que a partir de que ciertas ecuaciones son retenidas durante cambios teóricos, estas ecuaciones nos digan algo sobre la estructura del mundo. Especialmente, no queda claro que tales ecuaciones representen relaciones reales entre entidades físicas que, de otra forma, serían incognoscibles. 
(III) Las descripciones sobre el mundo, obtenidas a partir de nuestras teorías empíricas, no se pueden expresar únicamente con ecuaciones, también se requieren algunas presunciones teóricas que no forman parte de éstas para justificar el éxito de una teoría empírica.

(IV) La distinción entre estructura y naturaleza de una entidad, como la hace Worrall, no se puede justificar. Según Psillos, los científicos normalmente describen la naturaleza de una entidad atribuyéndole ciertas propiedades y relaciones. El comportamiento nomológico de ésta se describe entonces mediante ecuaciones.

Una pregunta abierta para el realismo estructural, formulada por Frigg y Votsis (2011), busca aclarar cómo es la relación formal de correspondencia entre dos estructuras. Si una de éstas es retenida y reaparece en una teoría nueva, se debe mostrar en qué consiste la correspondencia entre la estructura de la teoría antecesora y la teoría nueva.

\section{Ampliando y fortaleciendo el realismo estructural}

Hasta este punto he introducido el realismo estructural y recordado las objeciones que se le han hecho. En esta parte expondré cómo éste puede ser ampliado, no sólo aludiendo a ecuaciones, sino pensando en estructuras en un sentido más amplio. Para ello, primero introduciré el marco conceptual de la metateoría estructuralista, dentro del cual, propongo, debe efectuarse la formulación del realismo estructural. Luego mostraré cómo pueden ser respondidas las objeciones que se le han hecho.

La metateoría estructuralista es una concepción modelo-teórica de teorías empíricas. Exposiciones canónicas se encuentran en Balzer, Moulines, Sneed (1987), y en Díez, Moulines (1997). Se caracterizan las teorías en términos de teoría de modelos, usando esta herramienta matemática. En esta concepción, una teoría empírica consiste de sus modelos, son secuencias de la siguiente forma:

$$
<D_{1} \ldots, D_{m}, R_{1} \ldots, R_{n}>
$$


Las $D_{1}$ son los conjuntos básicos y las $R_{1}$ son relaciones construidas sobre estos conjuntos. Los $D_{1}$ contienen lo que se toma por la ontología de la teoría, los "objetos" que son asumidos como "reales" por la teoría. Los $R_{1}$ son funciones. En teorías empíricas que hacen uso de herramientas cuantitativas, son normalmente funciones que proyectan objetos empíricos a los números reales. Una teoría empírica consiste de los siguientes conjuntos de modelos:

(I) La primera parte es el núcleo $K$, que consiste de la siguiene tupla: $K=<T, I>$. El así llamado elemento teórico $T$ es un conjunto de modelos que consiste en la unión de los modelos potenciales, $M_{p}$; modelos parciales potenciales, $M_{p} p$; modelos actuales, $M$; condiciones de ligaduras globales, $G C$, y vínculos interteóricos globales GL. Aparte de $T, I$ es el conjunto de aplicaciones intencionales.

(II) Un elemento teórico $T$ es a su vez una estructura compleja, tiene la forma de la siguiente tupla:

$$
T=<M_{p}, M_{p} p, M, G C, G L>
$$

El aparato técnico formal de la metateoría estructuralista se mostrará como adecuado para dar más fuerza al realismo estructural. En lo que sigue se dará primero una elucidación informal de lo que significa cada parte de un elemento-teórico y después se mostrará de qué manera se puede fortalecer el realismo estructural con este marco conceptual introducido y se verá un camino para responder a sus objeciones.

Un conjunto de modelos potenciales $\left(M_{p}\right)$ fija el marco conceptual general, dentro del cual se caracteriza un modelo actual de una teoría. Todas las entidades que se pueden subsumir bajo el mismo marco conceptual de una teoría dada son miembros de los conjuntos de modelos potenciales de esta teoría. Conjuntos de modelos parciales potenciales $\left(M_{p} p\right)$ representan el marco conceptual de datos para la corroboración o refutación de una teoría. Los conceptos en $M_{p} p$ se pueden determinar independientemente de $T$. Esto significa que términos que son teóricos en los conjuntos de modelos potenciales de una teoría se dejan afuera, dado que los $M_{p} p$ son los modelos de datos. Los conjuntos 
de modelos que no sólo pertenecen al mismo marco conceptual, sino que también satisfacen las leyes de la misma teoría son los conjuntos de modelos actuales $M$. Dado que aplicaciones locales de una teoría se solapan en el espacio y en el tiempo, se requieren los conjuntos de condicions de ligadura globales $G C$ para caracterizarlo. Mediante esta herramienta se expresan los requisitos formales que restringen las componentes de un modelo en dependencia de otras componentes de otras modelos. Estas condiciones de ligadura expresan conexiones físicas entre diferentes aplicaciones de una teoría, son sus relaciones intrateóricas. Los conjuntos de vínculos interteóricos globales GL representan las conexiones entre teorías diferentes, dado que ciertas entidades y leyes pueden ser parte de teorías empíricas diferentes.

En lo que sigue, expondré una parte de una recostrucción estructuralista de la mecánica clásica de choques, contenida en Balzer, Moulines, Sneed (1987, pp. 26-27; 96-97). Esto con el fin de que el lector tenga un ejemplo claro de cómo se aplica la metateoría estructuralista en un caso concreto. Las colisiones se describen, marcando la velocidad de cada partícula antes y después de la colisión. Nada se dice sobre el fenómeno de la colisión en sí. En lo siguiente, se muestra la definición del modelo potencial $M_{p}$ de este elemento teórico:

$M C C: x$ es una mecánica clásica de choque potencial $\left(x \in M_{p}(C C M)\right)$ si y sólo si, hay: $P, T, R, v, m$, tal que:

(1) $x=P, T, R, v, m$;

(2) $P$ es un conjunto finito, no-vacío;

(3) $T$ contiene exactamente dos elementos $\left(T=t_{1}, t_{2}\right)$;

(4) $v: P \times T \rightarrow R^{3}$;

(5) $m: P \rightarrow R^{+}$.

$P$ es un conjunto de cuerpos discretos (partículas), $T$ es un conjunto de instantes de tiempo. $T$ contiene dos puntos del tiempo. La función $v$ es la función de velocidad. Esta función asigna a cada partícula $p$ en un punto de tiempo $t$, su velocidad $v(p, t)$ como elemento del conjunto de números reales $R^{3}$. La velocidad es una función vectorial que depende del tiempo, su codominio son los números reales. Esta función asigna un vector de tres componentes (para cada dirección en el espa- 
cio) a cada partícula en cada instante del tiempo. $m$ es la función de masa. Esta función asignoa a cada partícula su masa $m(p)$ que, según (5), debe ser positiva. La función de masa es una función escalar independiente del tiempo, su codominio son los números reales.

En la metateoría estructuralista, se asume que después de una recostruccuón lógica - con el aparato formal aquí introducido- de nuestras teorías empíricas, se obtiene como resultado, aparte de la estructura lógica compleja de la teoría, resultados sobre sus relaciones (los vínculos interteóricos) con otras teorías. Dentro de estas relacions, se puede identificar estructuras que aparecen en ambas teorías relacionadas. Los respectivos modelos potenciales de las teorías se pueden relacionar mediante tales relaciones. De esta manera, se puede hacer explícita la noción de continuidad estructural dentro del marco conceptual de la metateoría estructuralista. Esto es sólo una alusión. Pretendo exponer aquí que cualquier término técnico de la metateoría estructuralista que siver para modelar conexiones entre teorías (como el de vinculación interteórica) sirve para modelar en cada caso específico cómo se ve exactamente la continuidad entre dos teorías. Además, si entendemos nuestras teorías empíricas como estructuras, tal como es el caso en la metateoría estructuralista, se sigue de esto que cualquier conexión enre dos teorías será una conexión estructural, esto trivialmente. Quiero mostrar que mediante estudios de casos concretos, reconstruidos con el aparato de la metateoría estructuralista, se pueden modelar de la manera más exacta las relaciones entre teorías empíricas, estas relaciones pueden contar como relaciones de continuidad estructural, en el sentido del realismo estructural.

En contra de mi propuesta se puede argumentar que del hecho de que se puedan mostrar formalmente las relaciones interteóricas no se sigue que estas estructuras sean de alguna forma "reales". Es aquí donde quiere recordar lo que he formulado como las dos presunciones metafísicas en las que se basa el realismo estructural, en la parte III de este trabajo. Si aquellas estructuras permanecen una y otra vez más mediante cambios teóricos radicales, esto nos quiere decir que "algo" de nuestra representación estructural refleja el mundo de manera correcta.

En el trabajo de Sneed (1983) se pueden ver semejanzas con el realismo estructural, aunque la metateoría estructuralista nunca ha sido 
formulada explícitamente como una posición dentro del debate sobre el realismo científico. En este trabajo Sneed nos dice lo siguiente:

Es importante entender que, en la vista estructuralista, teorías empíricas hacen aserciones concretas, descriptivas y falsificables sobre su objeto de estudio. El estructuralismo no es solamente una versión nueva de instrumentalismo, escondiéndose detrás de una nube de notación conjuntista. Del otro lado, los estructuralistas ven las formulaciones matemáticas asociadas con una teoría como partes mucho más esenciales de la teoría que las aserciones que ella hace. Las aserciones pueden cambiar durante el desarrollo histórico de la teoría, pero el aparato matemático permanece igual. Así, ambos, el estructuralista y el realista afirmarían que la ciencia empírica hace aserciones descriptivas, pero estarían en desacuerdo hasta donde los científicos empíricos, hablando profesionalmente, "piensan lo que dicen" (Sneed, 1983, p. 351).

En esta cita de Sneed, quien es uno de los fundadores de la metateoría estructuralista, se encuentra claramente una semejanza a las posturas de Worrall. Aunque Sneed no habla de una continuidad estructural y de una retención de ciertas ecuaciones, dice explícitamente que el aparato matemático de una teoría permanece igual. Es ahí donde veo una relación estrecha entre las propuestas de los dos autores. Aunque la metateoría estructuralista ha sido formulada principalmente como un programa para dar cuenta de la estructura lógica de nuestras teorías empíricas, de la dinámica de la ciencia y dado que se piensa como una postura neutral a debates sobre el realismo, ${ }^{2}$ pienso que la aceptación de su marco conceptual como instrumento de reconstruccuón lógica de nuestras teorías empíricas, combinado con las dos presunciones metafísicas expuestas arriba, lleva a una forma de realismo estructural; este, se puede ver fortalecido de manera significativa, si adopta el marco conceptual de la metateoría estructuralista.

Para responder a las objeciones que se le han hecho al realismo estructural, se puede proceder de la siguiente manera:

Respuesta a las objeciones (1), (2) y (3): La crítica de Psillos tiene razón en parte. Es justo por esto que se debe ampliar la noción de continuidad estructural a algo más que sólo ecuaciones. Con esta crítica, Psillos posibilita un cambio de enfoque como lo propongo

\footnotetext{
${ }^{2}$ En conversación personal, esto me han dicho tanto Ulises Moulines, como Wolfgang Bal-
} zer. Ambos son cofundadores del programa estructuralista. 
yo en este trabajo. No sólo las ecuaciones son continuas, sino ciertas partes de nuestras teorías, caracterizadas en términos de la metateoría estructuralista, dependiendo en cada caso concreto de un estudio de caso. Ya que las teorías son identificadas como estructuras, en el sentido formal expuesto arriba, sus conexiones serán estructurales. Cuando una ecuación se retiene, es simplemente expresado por un vínculo interteórico de dos modelos potenciales o actuales de dos elementos teóricos. Ahí habrá que ver cada caso concreto, basado en su reconstrucción lógica concreta. No sólo las ecuaciones, sino la representación completa en términos de la metateoría estructuralista, nos da una representación adecuada de la estructura lógica de la teoría empírica reconstruida. Aquella teoría, a su vez, nos da una representación de ciertos fenómenos de los que trata. No sólo las ecuaciones, sino toda la teoría, entendida como estructura, nos da una representación estructural del mundo. Las tres objeciones de Psillos se pueden responder de esta forma.

Respuesta a (4): La distinción entre estructura y naturaleza de una entidad se puede defender para el realismo estructural, refiriendo al trabajo de Carnap (1928), donde muestra la necesidad de la misma:

Cada enunciado científico puede en principio ser transformado de tal forma que es solamente un enunciado estructural. Pero esta transformación no es solamente posible, sino requerida. Porque la ciencia quiere hablar de lo objetivo; como sea, todo lo que no pertenece a la estructura, pero a lo material, todo lo que es señalado concretamente,es al final subjetivo. Desde el punto de vista de la teoría de la constitución, este asunto debe ser expresado de la siguiente forma. La serie de experiencias es diferente para cada sujeto. Si buscamos, en este sentido, un acuerdo para los nombres de los objetos, constituidos en la base de experiencias, entonces, esto no puede suceder por medio de la referencia al material completamente divergente, sino sólo puede pasar por los indicadores formales de las estructuras de los objetos $(\$ 16)$.

Si adoptamos el criterio de Carnap para descripciones estructurales, queda clara la motivación de una distinción entre naturaleza y estructura de una entidad. Análogamente, la metateoría estructuralista proporciona descripciones estructurales de las teorías empíricas. Las entidades son descritas de manera estructural, no mediante alguna referencia, que, al final siempre será subjetiva. De esta forma, el realis- 
mo estructural no puede, ni tiene que comprometerse a alguna teoría de la referencia. Psillos, como parece, presupone que no hay nungún obstáculo cuando se realiza un acto de referencia y por esto sí le es posible comprometerse con una teoría así. ${ }^{3}$

Hacer uso de posiciones del Aufbau en el debate actual no es anacrónico, dado que trabajos recientes -Cfr. Moulines, 1996 y Leitgeb, 2006han mostrado que, si bien el programa originalmente expuesto por Carnap no ha podido ser realizado completamente, sin embargo, de manera restringida, sí es consistente la propuesta carnapiana original.

A su vez, cuestiones de correspondencia entre dos estructuras se resuelven "automáticamente", ya que dentro del marco conceptual de la metateoría estructuralista, cualquier relación entre ellas es expresable formalmente en términos de teoría de conjuntos.

Para ver con más detalle cómo puede ser una continuidad estructural en términos de la metateoría estructuralista, introduzco aquí el concepto de especialización. Éste, es parte del marco de la metateoríia estructuralista, definido en Balzer, Moulines y Sneed (1987, pp. 168172). Una especialización es una relación entre dos elementos teóricos, definida de lo siguiente:

Si $T=<M_{p}, M_{p} p, M, G C, G L, I>$ y $T^{\prime}=<M_{p}^{\prime}, M_{p} p^{\prime}, M^{\prime}, G C^{\prime}, G L^{\prime}, I^{\prime}>$ son elementos teóricos, entonces:

$T^{\prime}$ es una especialización de $T$, si:

(1) $M_{p}^{\prime}=M_{p}$

(2) $M_{p} p^{\prime}=M_{p} p$

(3) $M^{\prime} \subseteq M$;

(4) $G C^{\prime} \subseteq G C$;

(5) $G L^{\prime} \subseteq G L$;

(6) $I^{\prime} \subseteq I$.

La especialización expresa el hecho que nuestras teorías tienen aplicaciones más específicas a un sistema más local y más restringido. Dentro de esta aplicación, algunas de las leyes de la teoría en cuestión son

\footnotetext{
${ }^{3}$ Psillos sí ofrece una amplia y detallada exposición de lo que podría ser una teoría de la referencia contundente, en 1999, pp. 280-300. Dado que una discusión de esta posición y sus posibles relaciones con el realismo estructural llevarían demasiado lejos dentro del marco de este artículo, queda esto abierta para un trabajo futuro.
} 
utilizadas de manera más especial, o ciertas leyes auxiliares son aplicadas. Esto es expresado en la definición, donde en el punto (3) se expresa que los conjuntos de modelos actuales $M^{\prime}$ del elemento teórico $T^{\prime}$ son subconjunto de los conjuntos de modelos actuales $M$ del elemento teórico $T$. Dado que las leyes de una teoría son partes de los modelos actuales, esto expresa formalmente que las leyes del elemento teórico $T^{\prime}$ son más específicas que las de $T$. Mientras tanto, ambos elementos tratan de lo mismo, dado que sus marcos conceptuales generales, los modelos potenciales $\left(M_{p}\right)$, son idénticos. La siguiente cita ilustra la necesidad de la introducción del concepto de especialización:

Cuando consideramos nuestra reconstrucción de la mecánica clásica de partículas, el lector se puede haber preguntado ya dónde hemos dejado leyes tan importantes de la mecánica clásica de partículas como la tercera ley de Newton -el principio de actio-reactio-, la ley de la gravitación, o la ley de Hook. Nuestra respuesta es: todos ellos constituyen elementos teóricos diferentes de la mecánica clásica de partículas, pero sí están relacionados. Todo el orden, en cambio, constituye lo que llamamos la "red teórica de la mecánica clásica de partículas”. Lo mismo vale para otras teorías avanzadas de la ciencia empírica. En el caso de la termodinámica de simple equilibrio, aparte de la ecuación fundamental y las condiciones de ligadura y los vínculos interteóricos -los cuales, admitimos, proporcionan mucho del contenido de esta teoría-, uno quisiera ver el "tercer principio de la termodinámica" de Nernst, la ley de Gay-Lussac, y otras leyes más especiales. Muchas de estas leyes especiales de la teoría son, más bien, asociadas con condiciones de ligadura particulares y, posiblemente, vínculos interteóricos, aparte de los que ya se han explicado, cuando se trata de los elementos teóricos básicos. En otras palabras, la consideración de todos estos requisitos adicionales terminará en la reconstrucción de toda una serie de elementos teóricos diferentes, los cuales, como quiera, tienen la misma estructura básica. Por esta similitud de estructura podemos hablar de una red teórica y no sólo de un conjunto amorfo de elementos teóricos aislados y solitarios (Balzer, Moulines, Sneed, 1987, p. 168).

Es la misma ccomplejidad de las teorías empíricas que obliga a la introducción del concepto de red teórica y de especialización. Gráficamente, una red teórica se simboliza de la siguiente manera:

$T^{\prime}$ y $T^{\prime \prime}$ son los elementos teóricos más especializados, $T$ el más general. La continuidad estructural se obtiene entre $T$ y, respectivamente $T^{\prime}$ y $T^{\prime \prime}$. El ejemplo de las redes teóricas ilustra de manera ejemplar 


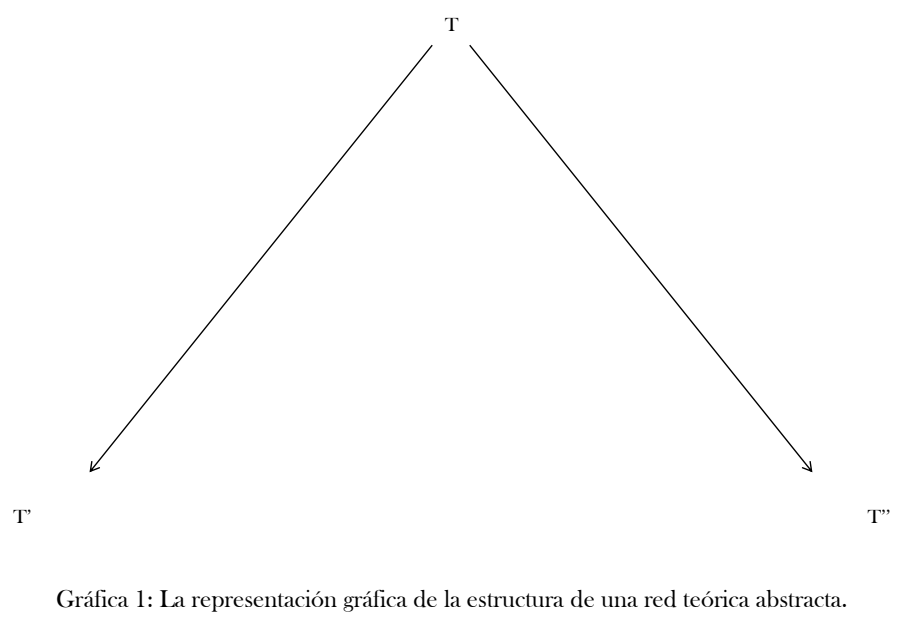

cómo es que haya continuidad entre dos estructuras. Cuando Worrall se refiere a la continua aparición de ciertas ecuaciones en diferentes teorías, la metateoría estructuralista nos proporciona una noción de continuidad estructural mucho más universal y abstracta. Esta noción es aplicable a elementos teóricos enteros; en su versión más abstracta, tiene la forma expuesta en la gráfica 1. En cada reconstrucción particular de una teoría, la red tendrá su forma específica, dependiento de las leyes de las teorías en cuestión. En este sentido, cuando una teoría tiene el suficiente desarrollo y grado de sofisticación, siempre se identificará alguna continuidad estructural en el sentido de especialización dentro de una red teórica. Que aquellas continuidades entre dos estructuras sean informativas, no triviales y que aclaren presunciones del realismo estructural dependerá de la forma concreta de cada red teórica en cada caso concreto.

El mencionar el fenómeno de especialización debe ser entendido sólo como un simple ejemplo con fines ilustrativos, para promover la idea de responder a preguntas del realismo estructural, aplicando la metateoría estructuralista. Desde luego debe quedar claro que el 
marco conceptual de la metateoría estructuralista ofrece toda una serie de conceptos ${ }^{4}$-como el de evolución, cristalización, holón-teórico, entre otros- mucho más complejos y ricos que pueden servir para modelar, clarificar y explicar la noción de continuidad estructural.

\section{Conclusión}

Mediante la exposición del surgimiento del realismo estructural dentro del debate sobre el realismo científico, mostré de qué manera este último ha sido introducido por Worrall. Se ha mencionado la serie de críticas que se le han formulado por parte de Psillos y otros. Pienso haber mostrado que la herramienta adecuada para caracterizar de manera más clara y precisa lo que es el realismo estructural es la metateoría estructuralista. Se ha visto que mediante la aplicación de ciertas nociones técnicas de esta concepción, la noción de continuidad estructural puede ser entendida de una manera más amplia. Esto sirve tanto para clarificar, como también para ampliar el realismo estructural. A la vez queda claro que sólo mediante estudios de casos concretos, las posturas del realismo estructural encontrarán su juicio donde se confirmarán o serán rechazadas. Aquellos estudios de caso deberán ser de cualquier disciplina de las ciencias empíricas, siempre y cuando sean teorías representativas las que se reconstruyen. En este sentido, no ha quedado fortalecido el realismo estructural directamente, pero se ha señalado un camino que puede ser recorrido por los realistas estructurales si buscan fortalecer y defender su posición, ya que la propuesta original de Worrall se ha visto demasiado susceptible a las críticas mencionadas. El realismo estructural como posición metafísica es defendible, de la mejor manera si se adapta el marco conceptual expuesto aquí.

\section{Referencias}

Balzer, W., C. U. Moulines y J. Sneed, 1987, An Architectonic for Science, Reidel, Dordrecht.

Boyd, R., 1983, "On the Current Status of the Issue of Scientific Realism" en Erkenntnis, vol. 19, pp. 45-90.

Carnap, R., 1928, Der ligische Aufbau der Welt Felix Meiner, Hamburgo.

${ }^{4}$ Expuestos igualmente en Balzer, Moulines, Sneed (1987). Recientemente, el trabajo de Moulines (2011) ofrece una serie de conceptos que parecen prometedores para el fin de modelar la noción de continuidad estructural. 
Cohen, L. J. y otros (eds.) Logic, Methodology and the Philosophy of Science VI, North-Holland Publishing Company, Ámsterdam.

Díez, J. y C. U. Moulines, 1997, Fundamentos de filosofía de la ciencia, Ariel, Barcelona.

Frigg, R. y I. Votsis, 2011, "Everything you Always Wanted to Know about Structural Realism but Were Afraid to Ask", en European Journal for Philosophy of Science, vol. 1, no. 2, pp. 227-276.

Ladyman, J., D. Ross, D. Spurrett y J. Collier, 2007, Every Thing Must Go: Metaphysics Naturalized, Oxford University Press, Oxford.

Laudan, L., 1981, "A Confutation of Convergent Realism", en Philosophy of Science, Vol. 48, no. 1, pp. 19-49.

Leitgeb, H., 2006, "A New Analysis of Quasianalysis", en Journal of Philosophical Logic, vol. 36, pp. 181-226.

Moulines, C. U., 1996, "Las Raíces Epistemológicas del Aufbau de Carnap", en El programa de Carnap. Ciencia, lenguaje, filosofía, C.E.L.C., Barcelona, pp. 45-74.

— 2011, "Cuatro tipos de desarrollo teórico en las ciencias empíricas", en Metatheoria. Revista de Filosofia e Historia de la Ciencia, vol. 1, no. 2, pp. 11-27.

Psillos, S., 1999, Scientific Realism: How Science Tracks Truth, Routledge, Londres.

Poincaré, Henri, 1912, La valeur de la science, Flammarion, París.

Russell, B., 1912, The Problems of Philosophy, Oxford University Press, Oxford.

—, 1927, The Analysis of Matter, George Allen \& Unwin, Londres.

Smart, J. J. C., 1963, Philosophy and Scientific Realism, RKP, Londres.

— 1979, "Difficulties for Realism in the Philosophy of Science", en Cohen y otros (eds.) 1979.

Sneed, J., 1983, "Structuralism and Scientific Realism”, en Erkenntnis, vol. 19, pp.245-370.

Worrall, J., 1989, "Structural Realism: The Best of Both Worlds?", en Dialectica, vol. 43, pp. 99-124.

Recibido el 14 de Junio de 2012

Aceptado el 28 de Junio de 2012 